\title{
Dosimetry of permanent interstitial prostate brachytherapy for an intraoperative procedure, using O-arm based CT and TRUS
}

\author{
Hiromichi Ishiyama, MD!, Akane Sekiguchi, MD!, Takefumi Satoh, MD², Hideyasu Tsumura, MD², Kouji Takenaka, MD!, \\ Shogo Kawakami, MD!, Ken-ichi Tabata, MD², Kentaro Kobayashi, MD², Masatsugu Iwamura, MD², \\ Kazushige Hayakawa, MD' \\ 'Department of Radiology and Radiation Oncology, ${ }^{2}$ Department of Urology, Kitasato University School of Medicine, Kitasato. \\ Sagamihara, Japan
}

\begin{abstract}
Purpose: The aim of this report is dosimetric evaluation for an intraoperative fusion computed tomography (CT) as a superior predictor of 1-month CT based dosimetry in comparison to transrectal ultrasound (TRUS) in permanent interstitial prostate brachytherapy.

Material and methods: Data of 65 patients treated with seed implantation were analyzed. All procedures has been performed with patients in the lithotomy position inside the $\mathrm{O}$-arm system. An end-fine probe is used as a landmark to fuse TRUS and O-arm-based CT images. There was no difference in the patient's position, probe position, and timing of image acquisition between the two imaging modalities. Dose-volume histogram (DVH) parameters such as the dose to $90 \%$ of prostate volume $\left(\mathrm{D}_{90}\right)$ has been analyzed.

Results: The area under the curve of the receiver operating characteristic tended to be larger on fusion CT than on TRUS for most DVH parameters $\left(71.85 \%\right.$ vs. $59.59 \%$ for $\left.\mathrm{D}_{90} ; p=0.07\right)$. Significant relationships between fusion $\mathrm{CT}$ and 1 -month CT were confirmed using Pearson's correlation coefficients for most DVH parameters $(R=0.48, p<0.01$ for $\left.\mathrm{D}_{90}\right)$, although the relationship between TRUS and 1-month CT was poor. Large dose reduction (35 Gy for $\left.\mathrm{D}_{90}\right)$ was seen from TRUS to fusion CT, especially in patients with high body weight and small prostate volume.

Conclusions: Intraoperative fusion CT appears to have higher predictive power for 1-month CT-based dosimetry than TRUS. A prospective trial using fusion CT-based planning is warranted.

Key words: brachytherapy, intraoperative $\mathrm{CT}$, low dose rate, $\mathrm{O}$-arm system, prostate cancer.

\section{Purpose}

Transrectal ultrasound (TRUS) is the standard imaging tool for interstitial prostate brachytherapy [1]. The prostate, urethra, and rectum are usually contoured on TRUS images, and treatment is planned based on these contours. In addition, these contours could be modified in a realtime manner during surgery. Meanwhile, it is a wellknown fact that ultrasound is not suitable for imaging implanted seeds [2,3]. Although computed tomography (CT) is not available in the usual operating room, the guideline of the American Brachytherapy Society recommended CT as the gold standard for detecting seed position and calculating post-implant dose volume histograms (DVHs) [4].

The O-arm ${ }^{\circledR}$ surgical imaging system (Medtronic, Dublin, Ireland) was developed to provide real-time, intraoperative CT imaging with a large field-of-view. This system permits patients to be in the lithotomy position even during image acquisition because the bore diameter of this system $(965 \mathrm{~mm})$ is significantly larger than that of conventional CT (700-800 mm). However, soft tissues such as the prostate or rectum are difficult to delineate with this system because of its lower contrast resolution compared to conventional CT, although high-density structures such as bone or seeds that are made from titanium can be clearly imaged.

Therefore, we combined O-arm-based CT and TRUS during surgery as a new strategy for intraoperative dosimetric evaluation. With this fusion dosimetry, we can compensate for the shortcomings of each modality because accurate seed position can be detected by CT images, and accurate contours can be delineated by ultrasound (US) images. In addition, highly matched fusion images can be expected since there is no difference in the patient's position, probe position, and timing of image acquisition between the two modalities.
Address for correspondence: Hiromichi Ishiyama, MD, Department of Radiology and Radiation Oncology, Kitasato University School of Medicine, 1-15-1 Kitasato, Sagamihara, Japan, phone: +81 7788453, fax: +81 7788906, 凶e-mail: hishiyam@kitasato-u.ac.jp
Received: 17.11 .2015

Accepted: 21.01.2016

Published: 26.02 .2016 
The purpose of this study was to evaluate whether fusion CT is better than TRUS for intraoperative dosimetry as a predictor of 1-month CT-based dosimetry.

\section{Material and methods}

\section{Patients}

The institutional review board approved this study (B15-01). Data of 65 patients treated with seed implantation during July 2014 to April 2015 were analyzed. Patients who received additional external beam radiotherapy were excluded. The patients' characteristics are shown in Table 1.

\section{Set-up}

After anesthesia, patients were placed in the O-arm system installed in our operating room. Zelefsky et al. have already reported the details of this system [5]. All procedures were performed with patients in the lithotomy position inside the $\mathrm{O}$-arm system. This position was kept throughout the whole operation, except when re-positioning was needed or in emergent situations.

\section{Image acquisition and implantation}

Transrectal ultrasound images of the whole prostate gland were acquired using a biplane transrectal ultrasound

Table 1. Patients' characteristics

\begin{tabular}{|c|c|}
\hline Age (y) & $69.3(6.6)$ \\
\hline \multicolumn{2}{|l|}{ T stage } \\
\hline $1 c$ & 33 \\
\hline $2 a$ & 15 \\
\hline $2 b$ & 7 \\
\hline $2 c$ & 10 \\
\hline iPSA (ng/ml) & $7.52(3.1)$ \\
\hline \multicolumn{2}{|l|}{ Gleason score } \\
\hline $3+3$ & 21 \\
\hline $3+4$ & 22 \\
\hline $3+5$ & 2 \\
\hline $4+3$ & 15 \\
\hline $4+4$ & 4 \\
\hline $4+5$ & 1 \\
\hline \multicolumn{2}{|c|}{ Hormonal therapy } \\
\hline Yes & 15 \\
\hline No & 50 \\
\hline Height (cm) & $165.3(5.8)$ \\
\hline Weight (kg) & $64.9(8.0)$ \\
\hline
\end{tabular}

probe (HI VISION Preirus, Hitachi Aloka Medical, Ltd., Tokyo, Japan). Peripheral needles were placed based on these $1^{\text {st }}$ TRUS images, and then $2^{\text {nd }}$ TRUS images were acquired after peripheral needle insertion. The prostate contour was modified based on the $2^{\text {nd }}$ TRUS images because of swelling and deformation of the prostate due to needle insertion.

All treatment plans were developed using Variseed software version 8.0.2 (Varian Medical Systems, Palo Alto, CA). The prescribed dose to the prostate with a 3- to 5 -mm margin was set at $145 \mathrm{~Gy}$. Two types of ${ }^{125} \mathrm{I}$ source (OncoSeed ${ }^{\circledR}$ model 6711, GE Health care Medi-Physics, Inc, Arlington Hts, IL, or BrachySource ${ }^{\circledR}$ model STM125I, CR BARD, Murray Hill, NJ) were used.

Both loose-seed and intraoperatively built customlinked (IBCL) seeds were used for patients in this study. Loose seeds were placed one by one transperineally through needles attached to a Mick applicator (Eckert \& Ziegler BEBIG, Berlin, Germany). Intraoperatively built custom-linked seeds were connected to each other using the Quick-link system (CR BARD, Murray Hill, NJ) and inserted through a relay system [6]. It has been reported that there is no dosimetric difference between loose seeds and IBCL seeds [7].

At the last phase of the operation, whole prostate contours of all slices were revised by the urologist and radiation oncologist, and matched to real-time prostate images through adjusting probe position, and then an end-fire probe was set at the most cranial slice of the prostate. Computed tomography images were then acquired using the O-arm system at $120 \mathrm{kV}, 50 \mathrm{~mA}$ and $200 \mathrm{mAs}$.

\section{Image fusion}

Acquired CT images were transferred to Variseed software. Figure 1 shows a representative case of image fusion technique. The end-fire probe was used as a landmark to fuse TRUS and O-arm-based CT images because it can be recognized in both modalities. In addition, a Foley catheter that was inserted in the urethra was visualized with both modalities and used for fine tuning. After image fusion, the contours of the prostate, urethra, and rectal wall were copied from TRUS to CT images. Therefore, the same contours were available on both modalities. A phantom study was done before data acquisition and confirmed fusion accuracy less than $1 \mathrm{~mm}$ deviation.

\section{Dose-volume histogram analysis}

Post-implanted CT analysis was done for all patients based on conventional CT images acquired 24 hours and 1 month after implantation. Urethral contouring was based on the outer rim of the urethral catheter, except for 1-month CT, in which the center of the prostate was used as a surrogate for urethral position. The rectal wall including sphincter muscle was fully contoured on 24-h and 1-month CT images, but only the anterior one-third excluding the lumen (body of the TRUS probe) was contoured on TRUS and fusion CT. The urethra and rectum were contoured in the same slices as the prostate contour.

Dose-volume histogram parameters including dose to $90 \%$ of prostate volume $\left(D_{90}\right)$, prostate volume receiv- 

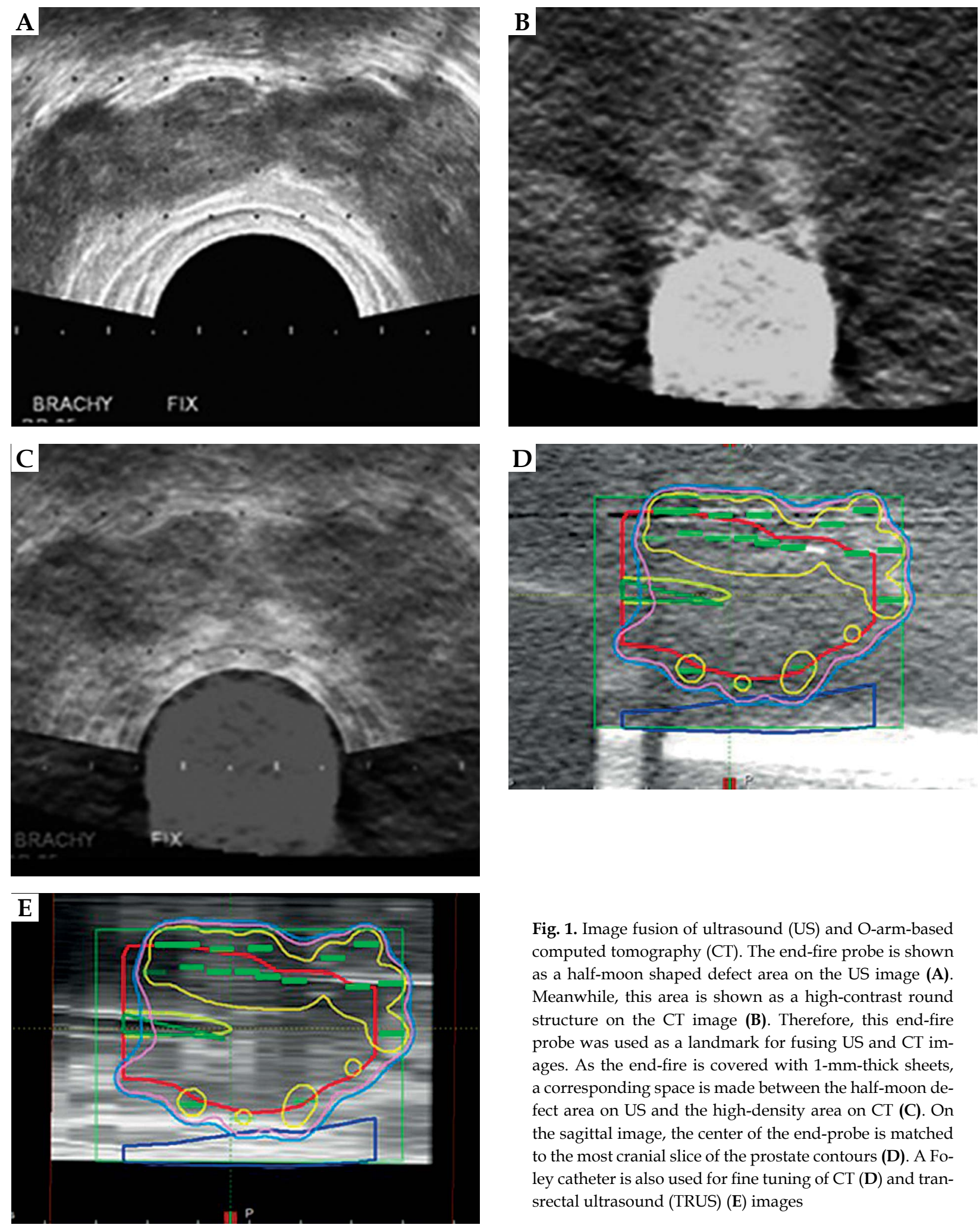

Fig. 1. Image fusion of ultrasound (US) and O-arm-based computed tomography (CT). The end-fire probe is shown as a half-moon shaped defect area on the US image (A). Meanwhile, this area is shown as a high-contrast round structure on the CT image (B). Therefore, this end-fire probe was used as a landmark for fusing US and CT images. As the end-fire is covered with 1-mm-thick sheets, a corresponding space is made between the half-moon defect area on US and the high-density area on CT (C). On the sagittal image, the center of the end-probe is matched to the most cranial slice of the prostate contours (D). A Foley catheter is also used for fine tuning of CT (D) and transrectal ultrasound (TRUS) (E) images

ing at least $100 \%$ dose $\left(\mathrm{V}_{100}\right)$, prostate volume receiving at least $150 \%$ dose $\left(\mathrm{V}_{150}\right)$, dose to $90 \%$ of urethral volume $\left(\mathrm{UD}_{90}\right)$, dose to $30 \%$ of urethral volume $\left(\mathrm{UD}_{30}\right)$, rectal volume receiving at least $100 \%$ dose $\left(\mathrm{RV}_{100}\right)$, and rectal volume receiving at least $150 \%$ dose $\left(R V_{150}\right)$ were collected from TRUS, fusion CT, 24-h CT, and 1-month CT.

\section{Statistical analysis}

Statistical analyses were performed using R software, version 3.2.0. Dosimetry of 1-month CT was defined as the gold standard [8]. Pearson's or Spearman's correlation coefficients were calculated for TRUS and fusion CT-based 
Table 2. Comparison between ultrasound- and computed tomography-based dosimetries

\begin{tabular}{lccccccccc} 
Parameter & TRUS & $p$ value & Fusion CT & $p$ value & $24-\mathrm{h}$ CT & $p$ value & 1-month CT & $p$ value \\
\hline $\mathrm{D}_{90}(\mathrm{~Gy})$ & $191(14.1)$ & $<0.001$ & $156(14.4)$ & $<0.001$ & $163(13.9)$ & $<0.001$ & $183(17.8)$ & 0.004 \\
\hline $\mathrm{V}_{100}(\%)$ & $98.8(1.50)$ & $<0.001$ & $93.0(4.51)$ & 0.002 & $95.3(5.90)$ & $<0.001$ & $97.6(3.27)$ & 0.004 \\
\hline $\mathrm{V}_{150}(\%)$ & $74.6(7.97)$ & $<0.001$ & $49.6(9.03)$ & n.s. & $50.5(10.4)$ & $<0.001$ & $68.8(11.5)$ & $<0.001$ \\
\hline $\mathrm{UD}_{90}(\mathrm{~Gy})$ & $174(17.1)$ & $<0.001$ & $134(17.4)$ & 0.002 & $143(21.7)$ & $<0.001$ & $172(22.7)$ & n.s. \\
\hline $\mathrm{UD}_{30}(\%)$ & $212(14.9)$ & $<0.001$ & $181(14.8)$ & n.s. & $178(15.7)$ & $<0.001$ & $212(18.9)$ & n.s. \\
\hline $\mathrm{RV}_{100}(\mathrm{cc})$ & $0.28(0.26)$ & $<0.001$ & $0.57(0.55)$ & 0.003 & $0.31(0.42)$ & $<0.001$ & $0.64(0.60)$ & $<0.001$ \\
\hline $\mathrm{RV}_{150}(\mathrm{cc})$ & $0.02(0.12)$ & $<0.001$ & $0.05(0.08)$ & n.s. & $0.04(0.07)$ & n.s. & $0.06(0.11)$ & $<0.001$
\end{tabular}

Values are given as means (standard deviation). Each $p$ value between columns corresponds to comparison of the two columns. $P$ value on right edge corresponds to comparison of 1-month CT and TRUS.

$D_{90}$-dose to $90 \%$ of prostate volume, $V_{100}$ - prostate volume receiving at least $100 \%$ of prescription dose, $V_{150}$ - prostate volume receiving at least $150 \%$ of prescription dose, $U D_{90}$ - dose to $90 \%$ of urethral volume, $U D_{30}$ - dose to $30 \%$ of urethral volume, $R V_{100}$ - rectal volume receiving at least $100 \%$ of prescription dose, $R V_{150}$ - rectal volume receiving at least $150 \%$ of prescription dose

TRUS - transrectal ultrasound, CT - computed tomography, n.S. - not significant

dosimetry in relation to the gold standard on 1-month CT. In addition, the diagnostic accuracies of TRUS and fusion CT to predict dosimetry based on 1-month CT were compared using receiver operating characteristic (ROC) analysis. Parameters of $\mathrm{D}_{90}>170 \mathrm{~Gy}$ and $\mathrm{V}_{100}>95 \%$, $\mathrm{V}_{150}<65 \%$ and $\mathrm{RV}_{100}<1$ cc on 1-month CT were defined as the reference for "good quality implant", although there is no validated definition.

The paired $t$-test was used to compare the DVH parameters of each modality. Risk factors related to the degree of discrepancy of $D_{90}$ between TRUS and fusion CT were assessed using Pearson's correlation coefficient.

\section{Results}

Table 2 shows dosimetric results based on TRUS, fusion CT, 24-h CT, and 1-month CT images. Significant differences between TRUS and fusion CT were detected, although the contours were the same. All DVH parameters except the rectum were significantly decreased on fu-

Table 3. Risk factors for a discrepancy in $\mathrm{D}_{90}$ (dose to $90 \%$ of prostate volume) between transrectal ultrasound (TRUS) and fusion computed tomography (CT)

\begin{tabular}{lcccc} 
Parameter & $\begin{array}{c}\text { Correlation } \\
\text { coefficient }\end{array}$ & $95 \% \mathrm{Cl}$ & $p$ value \\
\hline Weight & 0.31 & 0.07 & 0.51 & 0.013 \\
\hline Prostate volume* $^{*}$ & -0.82 & -0.97 & -0.18 & 0.024
\end{tabular}

*Measurement before implantation sion CT compared to TRUS. Surprisingly, the value of $\mathrm{D}_{90}$ dropped by 35 Gy from TRUS to fusion CT. Table 3 shows patient-related risk factors that had a significant effect on the degree of discrepancy between the two. Interestingly, patients with high body weight and small prostate volume tended to have a larger discrepancy between TRUS and fusion CT. As a matter of fact, DVH parameters that indicate a high dose on TRUS and a low dose on fusion CT were also detected as risk factors for a large discrepancy between TRUS and fusion CT (data not shown). Dose-volume histogram parameters of the prostate and urethra increased gradually from fusion CT through 24-h CT to 1-month CT. Meanwhile, DVH parameters of the rectum dropped temporarily on 24-h CT but increased again on 1-month CT (Table 2).

Table 4 shows the changes in the volumes of each structure. Volumes on TRUS and fusion CT were well matched because the same contouring was used. Meanwhile, rectal volume on 24-h CT was significantly larger than that of TRUS-based volume since contouring methods were different.

Figure 2 shows a representative case demonstrating discrepancies among TRUS, fusion CT, 24-h CT, and 1-month CT. Although seeds seemed to be implanted inside the prostate on TRUS images, corresponding seeds were implanted outside of the prostate on fusion CT images. Interestingly, on 24-h CT, prostate contouring was affected to a large degree by implanted seeds because of the "seeds-must-be-inside-the-prostate" bias. Prostate volume was slightly decreased on 1-month CT compared to $24-\mathrm{h} \mathrm{CT}$.

Table 4. Comparison of volumes of each structure between modalities

\begin{tabular}{lccccccccc} 
Parameter & \multicolumn{2}{c}{ TRUS } & \multicolumn{2}{c}{ Fusion CT } & \multicolumn{2}{c}{ 24-h CT } & \multicolumn{2}{c}{ 1-month CT } \\
\hline Prostate volume (cc) & 32.53 & 9.96 & 32.55 & 9.96 & 33.34 & 9.25 & 28.13 & 7.38 \\
\hline Urethral volume (cc) & 0.74 & 0.16 & 0.73 & 0.15 & 1.27 & 0.28 & 0.36 & 0.05 \\
\hline Rectal volume (cc) & 8.49 & 2.24 & 8.50 & 2.23 & 25.91 & 11.32 & 22.85 & 7.41
\end{tabular}

Values are given as means (standard deviation)

TRUS - transrectal ultrasound, CT - computed tomography 

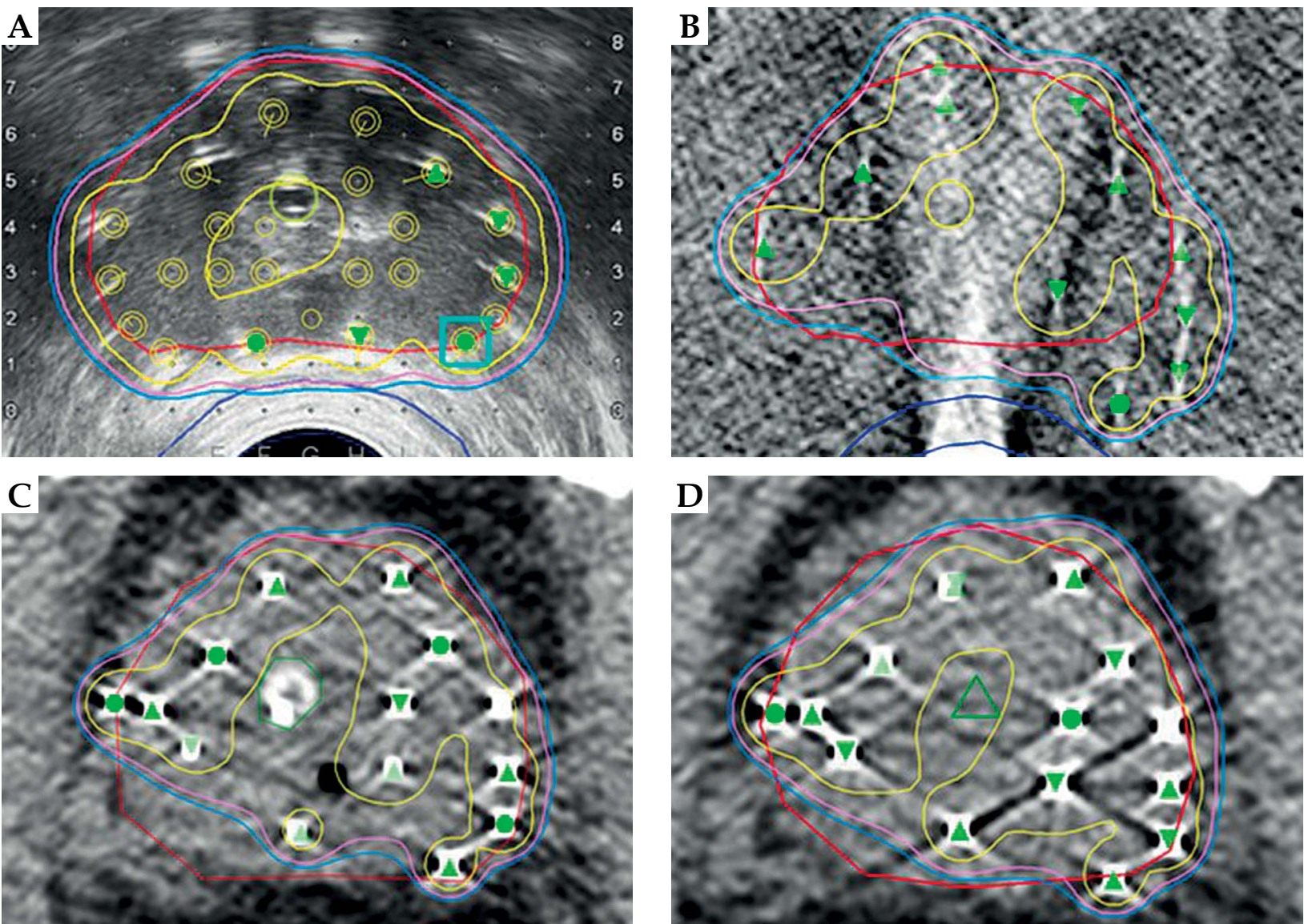

Fig. 2. Discrepancies among transrectal ultrasound (TRUS), fusion computed tomography (CT), 24-h CT, and 1-month CT-based dosimetries. Although most seeds seem to be implanted inside the prostate capsule on TRUS images (A), fusion CT shows that at least 4 seeds have been implanted outside the capsule (B). Due to streaking artifact from high-density parts of the probe, seed detection in the posterior-center part of prostate is difficult on fusion CT images (B). Therefore, manual adjustments by investigators are needed in many cases. Interestingly, the prostate contour is highly affected by implanted seeds on the 24-h CT (C). It seems that investigators tend to contour the prostate including all seeds. The prostate contour is slightly reduced on 1-month CT, although seed positions have not moved. Red line - prostate, dark blue line - rectum, light green or green circle Foley catheter, green triangle - urethra, blue line - $145 \mathrm{~Gy}$, pink line - $160 \mathrm{~Gy}$, yellow line - 217.5 Gy

Figure 3 shows relationships among TRUS, fusion CT, and 1-month CT for prostate $\mathrm{D}_{90}, \mathrm{~V}_{100}$, and $\mathrm{V}_{150}$. Significant relationships were seen between fusion $\mathrm{CT}$ and 1-month $\mathrm{CT}$ for all parameters. Only $\mathrm{V}_{150}$ showed a relationship between TRUS and 1 month CT. $R$ values between fusion CT and 1-month CT were higher than between TRUS and 1-month CT.

Figure 4 shows relationships among TRUS, fusion CT, and 1-month CT for $\mathrm{UD}_{90}$ and $\mathrm{UD}_{30}$. There were significant relationships among TRUS, fusion CT, and 1-month $\mathrm{CT}$ for $\mathrm{UD}_{30}$. $R$ values between fusion $\mathrm{CT}$ and 1-month CT were higher than between TRUS and 1-month CT. There were no significant relationships among any modalities for $\mathrm{RV}_{100}$ and $\mathrm{RV}_{150}$ (Figure 5).

Figure 6 shows the areas under the ROC curves of TRUS and fusion CT for the defined thresholds on 1-month CT. Area under the curve (AUC) values tended to be higher on fusion CT than on TRUS, except for $\mathrm{V}_{100}$ but not significantly. Table 5 shows the threshold for each DVH parameter derived from ROC, and their diagnostic performance with the best accuracy for the defined good quality implantation.

\section{Discussion}

Several papers have reported the usefulness of intraoperative CT-based dosimetry for interstitial prostate brachytherapy $[5,9,10]$. However, they did not match the patient's body position, inserted probe position, timing of image acquisition, and contours between TRUS and intraoperative CT as in the present study. Steggerda et al. have already reported the same fusion technique with satisfactory accuracy (average deviation less than $1 \mathrm{~mm}$ ) [11]. Their paper, however, focused on the geometrical accuracy of fusion imaging of $\mathrm{CT}$ and TRUS and showed little information about DVH parameters.

The inaccuracy of TRUS-based dosimetry has been reported in several papers. Nag et al. compared intraoperative US dosimetry with postoperative CT dosimetry acquired a few hours after operation [12]. As in the present study, they reported $16 \%$ and $10 \%$ differences in $\mathrm{D}_{90}$ and $\mathrm{V}_{100}$, respectively, and significant underestimation of rectal dose. Igidbashian et al. also reported the poor predic- 

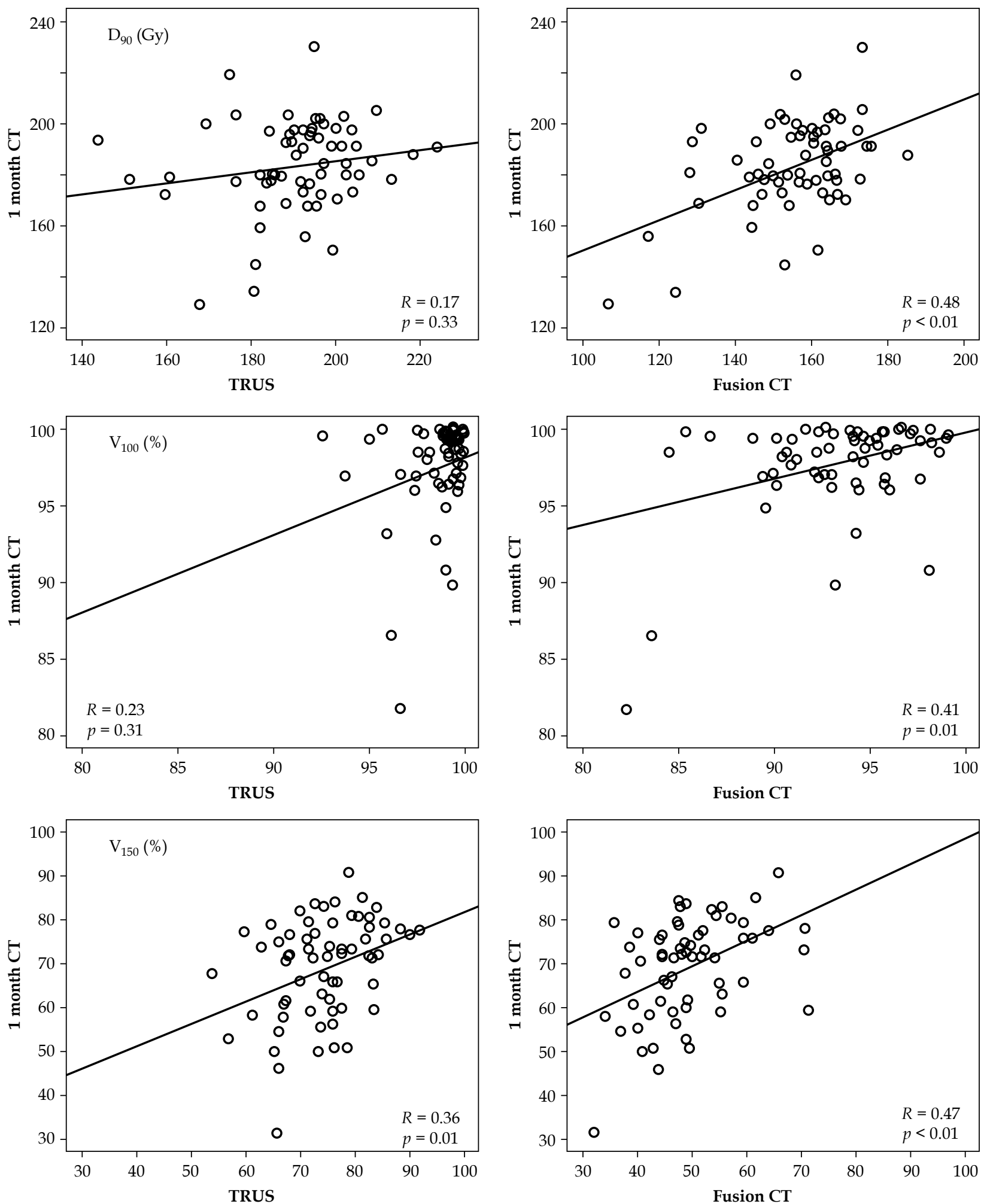

Fig. 3. Relationships among transrectal ultrasound (TRUS), fusion computed tomography (CT), and 1-month CT for prostate $\mathrm{D}_{90}, \mathrm{~V}_{100}$, and $\mathrm{V}_{150}$. The left column shows the relationship between TRUS and 1-month CT. The right column shows the relationship between fusion $\mathrm{CT}$ and 1-month CT. The regression line is also depicted in each figure. Significant relationships are seen between fusion CT and 1-month CT, although no relationship is seen between TRUS and 1-month CT, except $\mathrm{V}_{150}$. $R$ values between fusion $\mathrm{CT}$ and 1-month $\mathrm{CT}$ are higher than between TRUS and 1-month CT 

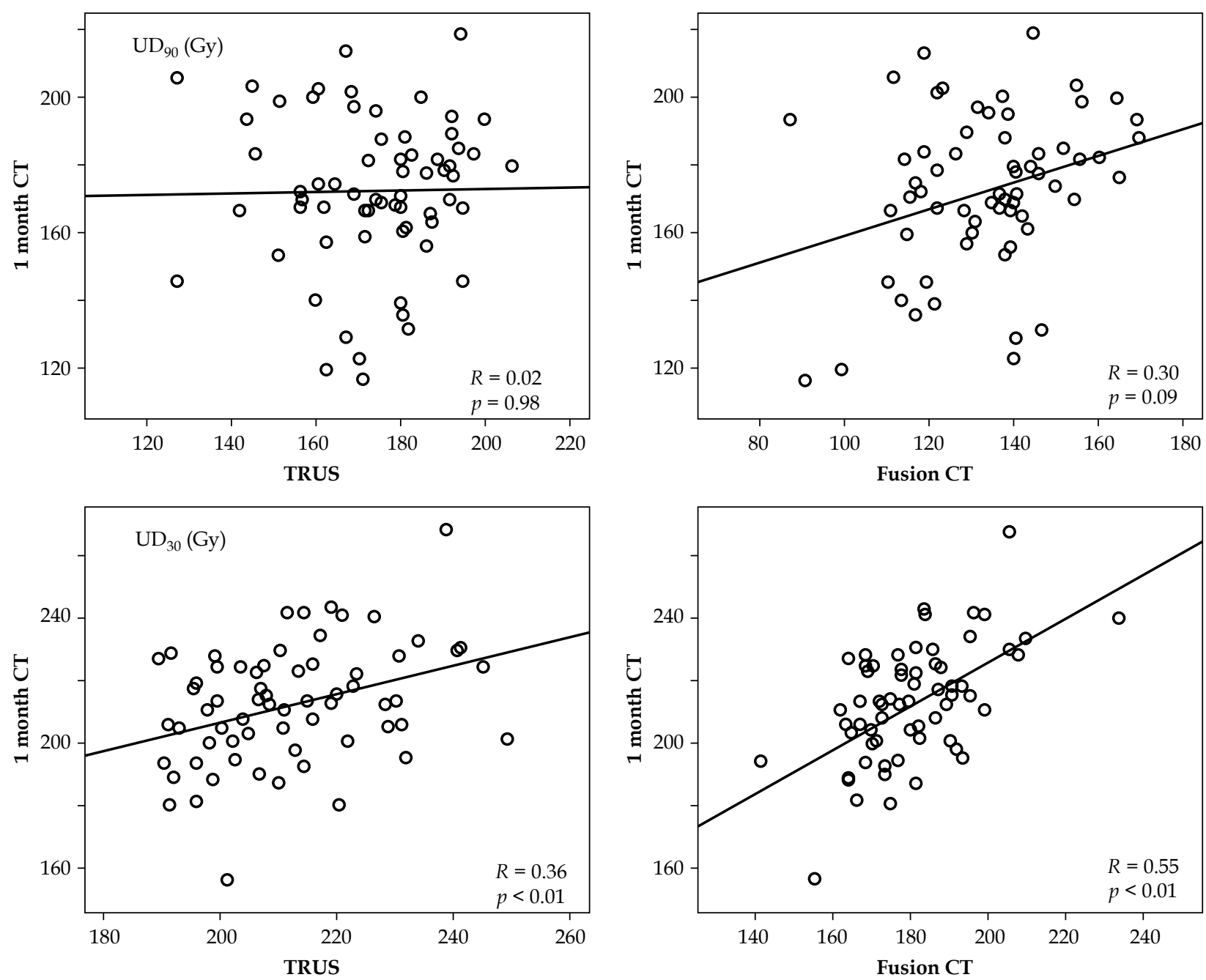

Fig. 4. Relationships among transrectal ultrasound (TRUS), fusion computed tomography (CT), and 1-month CT for UD 90 and $\mathrm{UD}_{30}$. Significant relationships are seen among the three modalities for $\mathrm{UD}_{30} . R$ values are higher between fusion $\mathrm{CT}$ and 1-month CT than between TRUS and 1-month CT

tive value of intraoperative TRUS dosimetry for 1-month CT-based dosimetry [13].

When comparing the dosimetries between TRUS and fusion CT, we can consider that the discrepancy was caused by "implant errors" because the contours of both modalities were the same. As shown in Figure 2, seed positions on TRUS were different from those on fusion CT. Especially for seeds in peripheral needles, they tended to unintentionally deposit in outer positions. This may be caused by obliquely inserted needles. Although the needle path was a completely horizontal line in the planning software (Variseed), most of the actual needles were obliquely inserted to varying degrees. Therefore, seed position would stray from the ideal path on the software to an unintentional position, especially for deeply implanted seeds. Patients with high body weight tended to have thick peritoneal soft tissue that would lengthen the distance between the template and the prostate. In addition, dose parameters such as $\mathrm{D}_{90}$ would be highly affected by implant errors when calculated from a small target volume. Therefore, the doses to the prostate and urethra were significantly reduced from TRUS to fusion CT, especially in patients with high body weight and small prostate volume. Doses to the rectum would be inversely increased because the seeds were implanted near the rectal wall in these cases.

When comparing dosimetries between fusion $\mathrm{CT}$ and 24-h CT, we can consider that the discrepancy was caused by "deformation" due to probe insertion and "contouring errors" of investigators, since seed positions and prostate volumes were probably not changing for 24 hours. As shown in Figure 2, prostate contours could be erroneously delineated by investigators. It has been reported that prostates are significantly larger on CT images than on MRI or US images [14,15], especially the seminal vesicles and apex. In addition, significant interobserver variation was also reported in prostate delineation on CT images $[16,17]$. Meanwhile, US images have a high degree of accuracy and a strong correlation with pathologic prostate volume [18]. In the present study, the value of $D_{90}$ and $\mathrm{V}_{100}$ increased slightly from fusion CT to 24-h CT. This was probably caused by erroneous delineation biased by seed position, as shown in Figure 2.

The area under the curve of TRUS was higher than that of fusion CT only for $\mathrm{V}_{100}$ in the present study (Figure 6). 

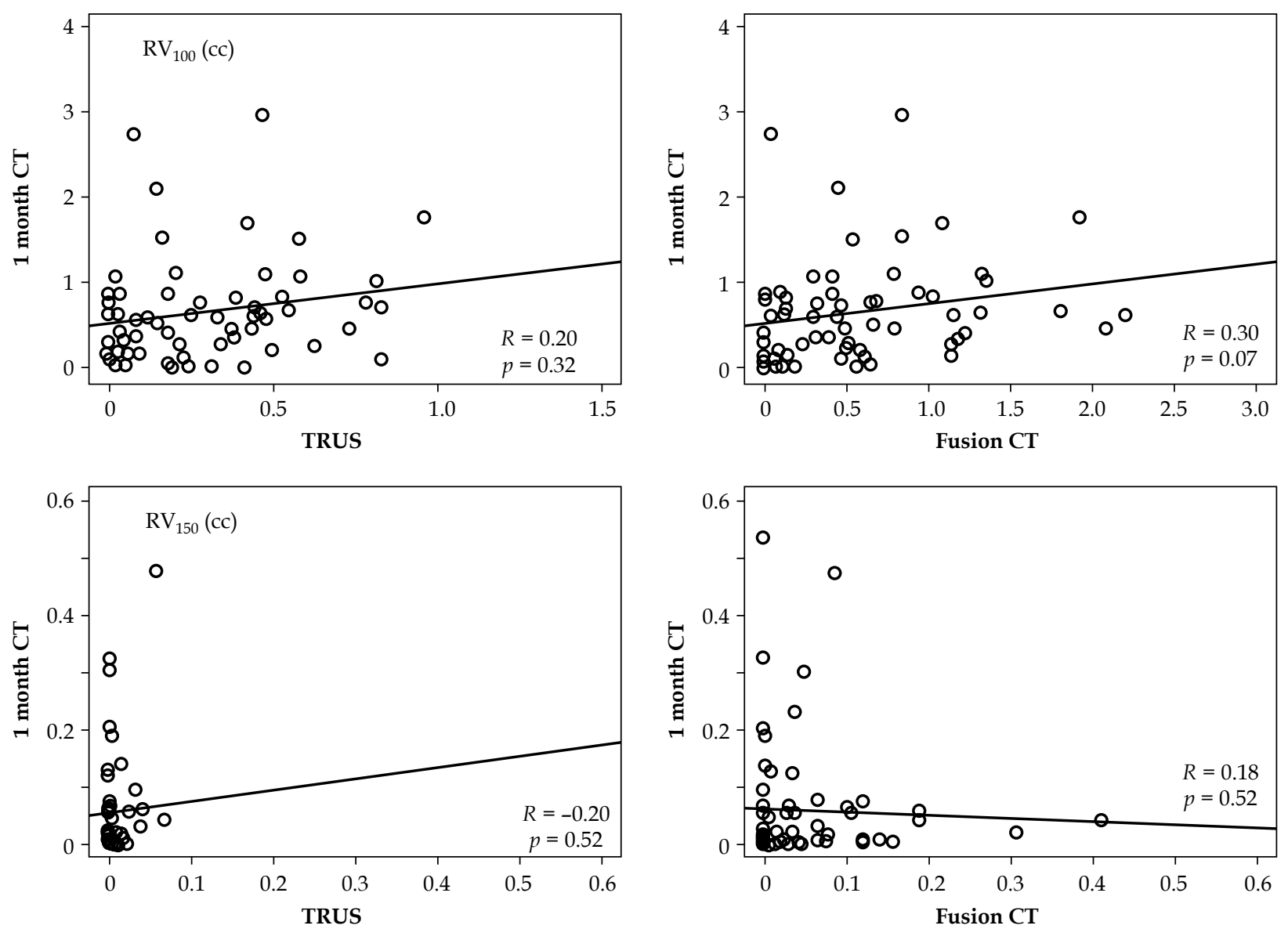

Fig. 5. Relationships among transrectal ultrasound (TRUS), fusion computed tomography (CT), and 1-month CT for RV $\mathrm{V}_{100}$ and $R V_{150}$. There are no significant relationships among any modalities for rectal $V_{100}$ and $V_{150}$, although the relationship between fusion $\mathrm{CT}$ and 1-month $\mathrm{CT}$ for $\mathrm{RV}_{100}$ has a relatively small $p$ value

This was probably caused by erroneous delineation on 1-month CT used as a standard. If the standard (1-month CT) was overestimated, the overestimating predictor (TRUS) would have higher prediction power compared to a more precise predictor (fusion CT). Prostate deformation caused by probe insertion may have some effects on $\mathrm{DVH}$, although its impact would be small, except for the rectal wall.

The value of $\mathrm{RV}_{100}$ decreased slightly from fusion $\mathrm{CT}$ to 24-h CT (Table 2). Though the anterior rectal wall on fusion CT was anteriorly deviated due to probe insertion, the deviation was resolved by removing the probe for 24-h CT. In addition, rectal contouring methods were different between the two modalities, as described above. These differences probably caused the DVH differences of $\mathrm{RV}_{100}$.

The current standard for postoperative dosimetric analysis was based on CT images after implantation, although recommended timing was not defined. However, it has been suggested that more than 30 days after implant would be needed to precisely evaluate the dose distribution, considering the decaying dose rate of ${ }^{125} \mathrm{I}$ and prostate volume change [8]. Therefore, we defined the 1-month CT as the gold standard. When comparing dosimetry between $24-\mathrm{h}$ CT and 1-month CT, we can consid- er that the discrepancy was caused by "volume change". Dose to every structure was significantly increased from 24-h CT to 1-month CT because of significant prostate volume reduction. Since the distances between the rectum and implanted seeds were reduced, dose to the rectal wall also increased on 1-month CT.

The present study showed that fusion CT had a higher predictive power for 1-month CT dosimetry than TRUS. Nevertheless, the predictive power of fusion CT was not satisfactory, as shown in Table 5. Sensitivity and specificity for predicting $\mathrm{D}_{90}>170$ Gy were only $67.9 \%$ and $75.0 \%$, respectively. Because 1-month CT images included not only implant errors but also contouring errors and volume changes, perfect prediction was probably impossible. At this time, we believe that reducing implant errors using fusion CT images is the best way to improve total implant quality. Especially for patients with high body weight and a small prostate, who tended to have large implant errors, as the present study suggested, fusion CT-based planning would be useful for high quality implantation.

The present study has several limitations. Prostate movement during TRUS image acquisition may cause some problems. Because mechanical shifting of the endfire probe from the base to the apex in a number of steps 

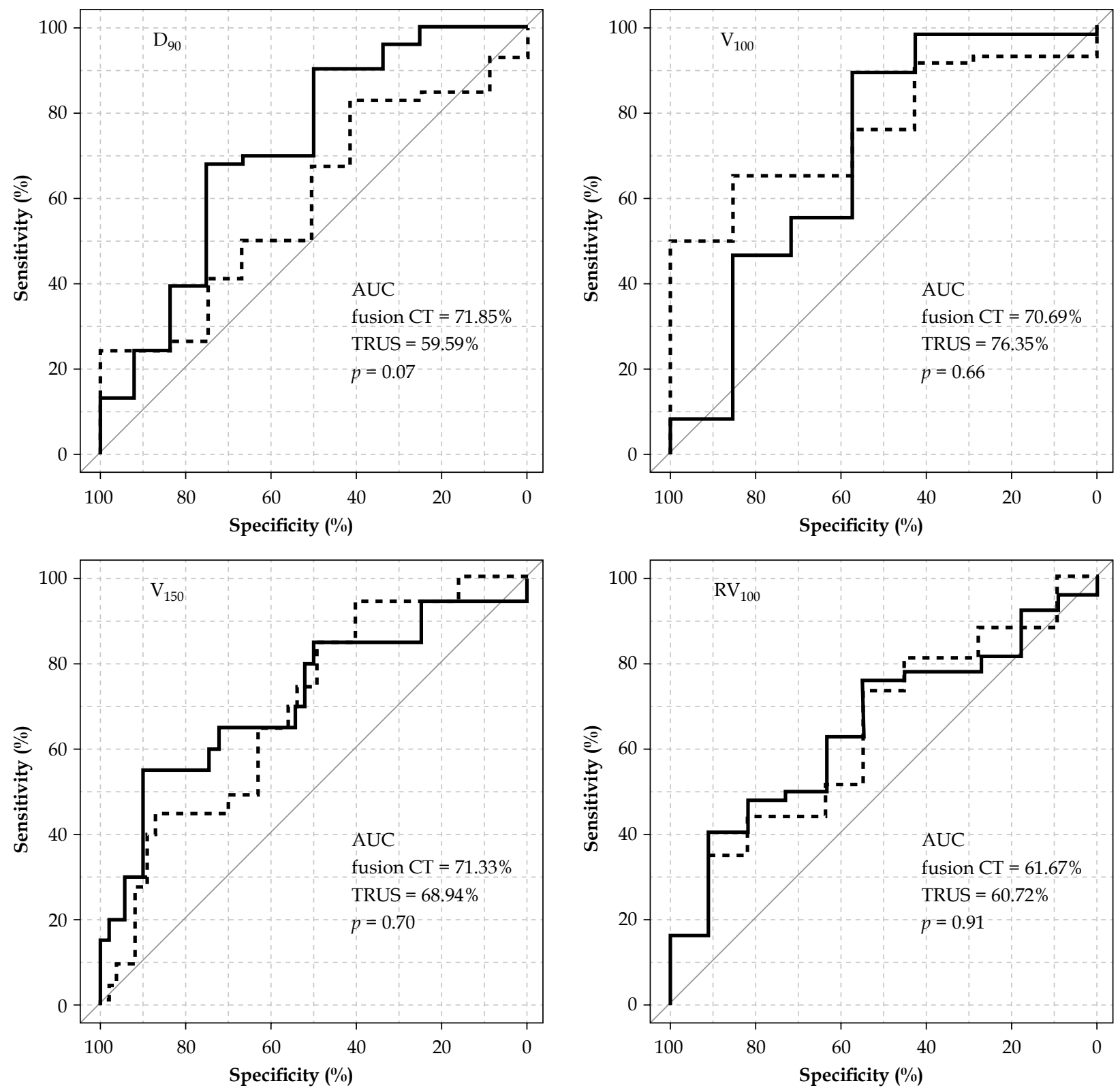

Fig. 6. Receiver operating characteristic curves for $\mathrm{D}_{90}, \mathrm{pV}_{100}, \mathrm{pV}_{150}$, and $\mathrm{RV}_{100}$. Area under the curve (AUC) values tend to be higher on fusion computed tomography (CT) than on transrectal ultrasound (TRUS), except for $\mathrm{V}_{100}$ but not significantly. Bold line - fusion CT, dotted line - TRUS

Table 5. Diagnostic performance with the best accuracy for good quality implant on 1-month computed tomography

\begin{tabular}{lcccccccc} 
& \multicolumn{2}{c}{$\mathrm{D}_{90}>170(\mathrm{~Gy})$} & \multicolumn{2}{c}{$\mathrm{V}_{100}>95(\%)$} & \multicolumn{2}{c}{$\mathrm{V}_{150}<65(\%)$} & \multicolumn{2}{c}{$\mathrm{RV}_{100}<1(\mathrm{cc})$} \\
\cline { 2 - 9 } & Fusion CT & TRUS & Fusion CT & TRUS & Fusion CT & TRUS & Fusion CT & TRUS \\
\hline Threshold & 154.7 & 183.2 & 89.9 & 99.1 & 44.5 & 78.9 & 0.26 & 0.42 \\
\hline Sensitivity (\%) & 67.9 & 83.0 & 89.7 & 65.5 & 55.0 & 95.0 & 40.7 & 74.1 \\
\hline PPV (\%) & 92.3 & 86.3 & 94.5 & 97.4 & 68.8 & 41.3 & 95.7 & 88.9 \\
\hline Specificity (\%) & 75.0 & 41.7 & 57.1 & 85.7 & 88.9 & 40.0 & 90.9 & 54.5 \\
\hline NPV (\%) & 34.6 & 35.7 & 40.0 & 23.1 & 81.6 & 94.7 & 23.8 & 30.0
\end{tabular}

CT - computed tomography, TRUS - transrectal ultrasound, PPV - positive predictive value, NPV - negative predictive value 
usually causes small prostate movement, reconstructed 3D images include not only prostate anatomy but also prostate movement. Therefore, contours on TRUS images may not perfectly match the actual prostate on CT images, because the probe was not moving during O-arm CT acquisition.

Another problem was streaking artifacts caused by seeds and radio-opaque parts inside the TRUS probe. Since the automatic seed-finding program of the planning software could not handle these strong artifacts perfectly, manual adjustment of seed detection was needed for almost all patients, although it took only 10 minutes.

The bore size of the O-arm system may be problematic for American or European people who are relatively taller than Japanese people. Although most of our patients could be in the lithotomy position, some patients who were relatively taller did not undergo CT due to positioning difficulty.

\section{Conclusions}

The present study showed that intraoperative fusion CT has higher predictive power for 1-month CT-based dosimetry than TRUS. In addition, the fusion CT-based dosimetry permits evaluation of "implant errors" without disturbance from contouring errors and volume changes. The present results also suggest that patients with high body weight and small prostate volume tended to have large implant error. To improve the quality of permanent interstitial prostate brachytherapy, especially for these high-risk patients, a prospective trial of fusion CT-based planning is warranted.

\section{Disclosure}

Dr. Ishiyama reports personal fees from Medicon, Inc., personal fees from Nihon Medi-Physics Co., Ltd., outside the submitted work.

Others authors report no conflict of interest.

\section{References}

1. Polo A, Salembier C, Venselaar J et al. Review of intraoperative imaging and planning techniques in permanent seed prostate brachytherapy. Radiother Oncol 2010; 94: 12-23.

2. Nag S, Bice W, DeWyngaert K et al. The American Brachytherapy Society recommendations for permanent prostate brachytherapy postimplant dosimetric analysis. Int J Radiat Oncol Biol Phys 2000; 46: 221-230.

3. Kirschner AN, Sathiaseelan V, Zhang Y et al. Multisector dosimetry in the immediate post-implant period: significant under dosage of the prostate base. J Contemp Brachytherapy 2014; 6: 33-39.

4. Davis BJ, Horwitz EM, Lee WR et al. American Brachytherapy Society consensus guidelines for transrectal ultrasound-guided permanent prostate brachytherapy. Brachytherapy 2012; 11: 6-19.

5. Zelefsky MJ, Worman M, Cohen GN et al. Real-time intraoperative computed tomography assessment of quality of permanent interstitial seed implantation for prostate cancer. Urology 2010; 76: 1138-1142.

6. Zauls AJ, Ashenafi MS, Onicescu G et al. Comparison of intraoperatively built custom linked seeds versus loose seed gun applicator technique using real-time intraoperative planning for permanent prostate brachytherapy. Int J Radiat Oncol Biol Phys 2011; 81: 1010-1016.

7. Ishiyama $\mathrm{H}$, Satoh $\mathrm{T}$, Kawakami $\mathrm{S}$ et al. A prospective quasi-randomized comparison of intraoperatively built custom-linked seeds versus loose seeds for prostate brachytherapy. Int J Radiat Oncol Biol Phys 2014; 90: 134-139.

8. Van Gellekom MP, Moerland MA, Kal HB et al. Biologically effective dose for permanent prostate brachytherapy taking into account postimplant edema. Int J Radiat Oncol Biol Phys 2002; 53: 422-433.

9. Kaplan ID, Meskell P, Oldenburg NE et al. Real-time computed tomography dosimetry during ultrasound-guided brachytherapy for prostate cancer. Brachytherapy 2006; 5: 147151.

10. Westendorp H, Hoekstra CJ, van't Riet A et al. Intraoperative adaptive brachytherapy of iodine- 125 prostate implants guided by $\mathrm{C}$-arm cone-beam computed tomography-based dosimetry. Brachytherapy 2007; 6: 231-237.

11. Steggerda M, Schneider C, van Herk M et al. The applicability of simultaneous TRUS-CT imaging for the evaluation of prostate seed implants. Med Phys 2005; 32: 2262-2270.

12. Nag S, Shi P, Liu B et al. Comparison of real-time intraoperative ultrasound-based dosimetry with postoperative computed tomography-based dosimetry for prostate brachytherapy. Int J Radiat Oncol Biol Phys 2008; 70: 311-317.

13. Igidbashian L, Donath D, Carrier JF et al. Poor predictive value of intraoperative real-time dosimetry for prostate seed brachytherapy. Int J Radiat Oncol Biol Phys 2008; 72: 605-609.

14. Rasch C, Barillot I, Remeijer P et al. Definition of the prostate in CT and MRI: a multi-observer study. Int J Radiat Oncol Biol Phys 1999; 43: 57-66.

15. Hoffelt SC, Marshall LM, Garzotto M et al. A comparison of CT scan to transrectal ultrasound-measured prostate volume in untreated prostate cancer. Int J Radiat Oncol Biol Phys 2003; 57: 29-32.

16. Al-Qaisieh B, Ash D, Bottomley DM et al. Impact of prostate volume evaluation by different observers on CT-based post-implant dosimetry. Radiother Oncol 2002; 62: 267-273.

17. Aoki M, Yorozu A, Dokiya T. Evaluation of interobserver differences in postimplant dosimetry following prostate brachytherapy and the efficacy of CT/MRI fusion imaging. Jpn J Radiol 2009; 27: 342-347.

18. Terris MK, Stamey TA. Determination of prostate volume by transrectal ultrasound. J Urol 1991; 145: 984-987. 